\title{
State-of-the-art CMR Mapping Techniques in the Detection of Subclinical Chemotherapy-induced Cardiotoxicity in Breast Cancer Patients
}

\author{
Noura Abdelmonem Atia ${ }^{1, ~ *, ~ L o r e n z ~ L e h m a n n ~}{ }^{2,7}$, Mohamed Adel Eltomey ${ }^{1}$, Mohamed Alm El-Din ${ }^{3}$, \\ Johannes Riffel ${ }^{2}$, Constantin Dreher ${ }^{4,5}$, Philipp Bäumer ${ }^{5}$, Anoshirwan Andrej Tavakoli ${ }^{5}$, \\ Norbert Frey $^{2}$, Heinz-Peter Schlemmer ${ }^{5}$, Matthias Friedrich ${ }^{2,6}$, Mahmoud Abdelaziz Dawoud ${ }^{1}$ \\ ${ }^{1}$ Department of Diagnostic Radiology, Faculty of Medicine, Tanta University, Tanta, Egypt \\ ${ }^{2}$ Department of Cardiology, Heidelberg University Hospital, Heidelberg, Germany \\ ${ }^{3}$ Department of Clinical Oncology, Faculty of Medicine, Tanta University, Tanta, Egypt \\ ${ }^{4}$ Department of Radiation Oncology, Medical Faculty Mannheim, Heidelberg University, Mannheim, Germany \\ ${ }^{5}$ DKFZ (German Cancer Research Center), Department of Radiology, Heidelberg, Germany \\ ${ }^{6}$ Departments of Medicine and Diagnostic Radiology, McGill University Health Centre, Montreal, Canada \\ ${ }^{7}$ DZHK (German Centre for Cardiovascular Research), Partner Site Heidelberg/Mannheim, Heidelberg, Germany
}

\section{Email address:}

noura.ateya@med.tanta.edu.eg (N. A. Atia)

${ }^{*}$ Corresponding author

\section{To cite this article:}

Noura Abdelmonem Atia, Lorenz Lehmann, Mohamed Adel Eltomey, Mohamed Alm El-Din, Johannes Riffel, Constantin Dreher, Philipp Bäumer, Anoshirwan Andrej Tavakoli, Norbert Frey, Heinz-Peter Schlemmer, Matthias Friedrich, Mahmoud Abdelaziz Dawoud. State-ofthe-art CMR Mapping Techniques in the Detection of Subclinical Chemotherapy-induced Cardiotoxicity in Breast Cancer Patients. International Journal of Medical Imaging. Vol. 9, No. 1, 2021, pp. 29-35. doi: 10.11648/j.jimi.20210901.13

Received: December 23, 2020; Accepted: December 30, 2020; Published: January 15, 2021

\begin{abstract}
Background: The oncological treatments have improved survival rates but with an increased risk of cardiovascular disease (CVD). Purpose: To detect subclinical cardiotoxic changes using Cardiovascular Magnetic Resonance (CMR) and to define parameters for the prediction of late cardiac changes after 3 months follow-up. Patients and methods: We conducted a prospective study in 21 breast cancer patients who were scheduled to undergo treatment either with anthracyclines, trastuzumab, or docetaxel. CMR scans were performed before therapy onset as well as 3-6 days and 3 months thereafter. Native left ventricular (LV) T1 and T2 parameters were acquired in addition to standard parameters. Results: Compared to baseline, the mean left ventricular ejection fraction (LVEF) tended to mildly decrease during follow-up. A significant reduction in mean native $\mathrm{T} 1$ was found from $1246.6 \pm 29.5 \mathrm{~ms}$ at baseline to $1231.4 \pm 31.4 \mathrm{~ms}$ at 3-6 days, which was followed by significant increase after 3 months reaching $1265.8 \pm 27.9 \mathrm{~ms}$ with $\mathrm{p}=0.011$ and 0.012 , respectively. A significant increase in mean T2 was also found from $41.6 \pm 3.4 \mathrm{~ms}$ at baseline to $43.8 \pm 3.8 \mathrm{~ms}$ after 3 months with $\mathrm{p}=0.045$. From 21 patients, only 1 patient $(4.8 \%)$ experienced cardiotoxicity. Conclusion: Treatment with potentially cardiotoxic drugs is associated with a change of CMR-derived native $\mathrm{T} 1$ which may enable an early identification of cardiotoxicity among breast cancer patients.
\end{abstract}

Keywords: Breast Cancer, Anthracyclines, Trastuzumab, Cardiotoxicity, T1 Mapping, Cardiac MRI

\section{Introduction}

Novel anti-cancer drugs have improved survival rates but at the cost of a significantly increased risk of CVD $[1,2]$.

Anthracyclines are known for relatively frequent cardiotoxic effects, which can be acute as myocarditis or chronic as ventricular dysfunction [3] or diffuse myocardial fibrosis [4]. Trastuzumab is one of the monoclonal antibody-based tyrosine kinase inhibitors $[5,3]$, with a demonstrated risk for ventricular dysfunction [6]. Docetaxel is a new anti-cancer drug that is suggested to induce LV diastolic dysfunction [7].

Until now, the proposed definitions rely on LVEF changes 
which are often subtle during initial phase of therapy [1] and do not include the early subclinical changes [3].

CMR has many advantages which may allow early identification of cardiotoxicity [8]. It offers the opportunity of quantitative myocardial tissue assessment using novel methods such as T1 and T2 mapping [9]. Diffuse myocardial fibrosis was suggested by a study which reported increase of native $\mathrm{T} 1$ several years after anthracycline therapy [4]. However, CMR has not yet been established as the primary method for monitoring patients treated with cardiotoxic chemotherapy [8].

The aim of this study was to identify patients at risk based on early detection of cardiotoxic changes using state-of-theart CMR techniques including T1 and T2 mapping and to identify parameters that could predict late cardiac changes after 3 months follow-up.

\section{Patients and Methods}

\subsection{Study Population}

This prospective observational longitudinal study was conducted during the period from April 2018 until June 2020. Twenty-one women diagnosed with breast cancer and scheduled to undergo treatment with anthracyclines, trastuzumab or docetaxel were enrolled. The inclusion criteria were: Age $\geq 18$ years, no previous start of chemotherapy. The exclusion criteria included: Pre-existing symptomatic HF, recent myocardial infarction, renal failure (GFR $\leq 30 \mathrm{ml} / \mathrm{min}$ ) and contraindications for magnetic resonance imaging.

\subsection{Preliminary Assessment}

All patients were subjected to medical history taking, clinical examination, 12-lead electrocardiography (ECG) and laboratory assessment including plasma $\mathrm{N}$-terminal pro brain natriuretic peptide (NT-proBNP) and serum high-sensitivity troponin $\mathrm{T}$ (hs-TnT) alongside CMR.

\subsection{Image Acquisition}

\subsubsection{Transthoracic Echocardiography (TTE)}

Was performed in all patients at baseline and $2^{\text {nd }}$ followup. The size and function of both ventricles were evaluated.

\subsection{2. $C M R$}

All enrolled patients performed three CMR examinations using $3 \mathrm{~T}$ Philips Ingenia, Best, Netherlands or $3 \mathrm{~T}$ Siemens MAGNETOM Prisma Fit, Erlangen, Germany equipped with body or cardiac coil and retrospective ECG gating. Scans were performed at baseline as well as 3-6 days and 3 months after chemotherapy onset. All patients were scanned using the previously published techniques. A breath-hold steadystate free precession was used to perform cine acquisitions with the following typical parameters: field of view $(\mathrm{FOV})=$ $350 \times 350 \mathrm{~mm}^{2}, \mathrm{TR} / \mathrm{TE}=2.8 / 1.4 \mathrm{~ms}$, slice thickness $=8 \mathrm{~mm}$, voxel size $=2.2 \times 2.2 \times 8 \mathrm{~mm}^{3}$, flip angle $(\mathrm{FA})=60^{\circ}$.

Native T1-mapping was performed using a breath-hold MOLLI sequence in three LV short axis (SAX) planes (basal, mid-ventricular and apical) with the following typical parameters: $\mathrm{FOV}=300 \times 300 \mathrm{~mm}^{2}, \mathrm{TR} / \mathrm{TE}=2.1 / 0.79 \mathrm{~ms}$, slice thickness $=10 \mathrm{~mm}$, voxel size $=2 \times 2 \times 10 \mathrm{~mm}^{3}$ and FA $=20^{\circ}$.

T2-mapping in three SAX planes as in T1-mapping. A breath-hold turbo SE sequence was used with the following typical parameters: $\mathrm{FOV}=350 \times 350 \mathrm{~mm}^{2}, \mathrm{TR}=1000 \mathrm{~ms}$, TE according to heart rate, slice thickness $=8-10 \mathrm{~mm}$, voxel size $=2 \times 2 \times 8 \mathrm{~mm}^{3}, \mathrm{FA}=90^{\circ}$.

Late gadolinium enhancement (LGE) images were acquired 10 minutes after intravenous (IV) injection of contrast agent (Gadovist ${ }^{\mathrm{TM}}$, Bayer Healthcare Pharmaceuticals, Bergkamen, Germany) at a dose of (0.1 $\mathrm{mmol} / \mathrm{kg}$ ) only at baseline to exclude a pre-existing myocardial scar. A breath-hold T1-weighted inversion recovery sequence was used with the following typical parameters: $\mathrm{FOV}=350 \times 350 \mathrm{~mm}^{2}, \mathrm{TR} / \mathrm{TE}=4.1 / 1.97 \mathrm{~ms}$, slice thickness $=8 \mathrm{~mm}$, voxel size $=1.79 \times 2.06 \times 8 \mathrm{~mm}^{3}$, $\mathrm{FA}=15^{\circ}$ and time of inversion $=250-425 \mathrm{~ms}$.

\subsection{CMR Analysis}

All images were analyzed using certified post-processing (cvi42 ${ }^{\mathrm{TM}}$ version 5.6.6, Circle Cardiovascular Imaging Inc., Calgary, Alberta, Canada). The size and function of both ventricles as well as LV mass index were quantitatively evaluated through manually tracing the epi- and endocardial borders on successive SAX cine images at end-diastole and end-systole. Papillary muscles and trabeculations were excluded from the blood volume. Ventricular wall motion abnormality was also assessed on cine images. Epicardial and endocardial contours were manually traced for $\mathrm{T} 1$ and $\mathrm{T} 2$ mapping in three SAX slices (basal, mid-ventricular and apical) with the application of a $10 \%$ safety margin to avoid partial volume effects. Two independent readers evaluated the LGE images in two perpendicular slices.

\subsection{Definition of Chemotherapy-related Cardiotoxicity}

According to the previously published criteria [10], chemotherapy-related cardiotoxicity was defined in this study as LVEF reduction of $\geq 5 \%$ to a value of $<55 \%$ when associated with HF symptoms or an asymptomatic LVEF reduction of $\geq 10 \%$ to a value of $<55 \%$.

\subsection{Statistical Analysis}

Data were analyzed using IBM ${ }^{\circledR}$ SPSS Statistics software version 26.0. All data are expressed either in mean \pm standard deviation (SD), median \& interquartile range (IQR) or frequency (number (n) - percentage (\%)). Repeated measures analysis of variance (ANOVA) and Friedman's test were used to evaluate temporal changes of continuous parametric and non-parametric variables, respectively with Bonferroni correction for multiple pairwise comparisons with baseline. Relationships between different variables were assessed using Pearson's correlation coefficient. The effect of various factors such as presence of CV risk factor, type or dose of chemotherapy used was tested by calculating the interaction with the CMR changes. Statistical significance was defined as a $p$ value $<0.05$. 


\section{Results}

\subsection{Patients Characteristics}

Table 1. Baseline characteristics

\begin{tabular}{ll}
\hline & Total (n=21) \\
\hline Age, (years) & $51.4 \pm 11.4$ \\
Body mass index $\left(\mathrm{kg} / \mathrm{m}^{2}\right)$ & $22.6 \pm 2.9$ \\
Patients using co-existing cardiac medication, n (\%) & $3(14)$ \\
Previous breast or mediastinal radiation, n (\%) & $2(9.5)$ \\
History of another tumor, n (\%) & $1(4.8)$ \\
Time from diagnosis until enrollment (months), Median (IQR) & $1(0-1)$ \\
Any cardiovascular risk factor, n (\%) & $16(76)$ \\
Hypertension, n (\%) & $3(14)$ \\
Dyslipidemia, n (\%) & $3(14.3)$ \\
Overweight or obesity, n (\%) & $6(28.6)$ \\
Smoking a, $\mathrm{n}(\%)$ & $8(38)$ \\
Family history of cardiovascular disease, n (\%) & $11(52.4)$ \\
Heart rate (beat/minute) & $72 \pm 11.8$ \\
\hline
\end{tabular}

Data are expressed as mean \pm SD and frequency ( $-\%)$ as well as median (IQR); ${ }^{\text {a }}$, either active smoking or previous history of smoking.

The baseline characteristics of the enrolled patients are listed in Table 1. One patient (4.8\%) had previous history of primary mediastinal B cell lymphoma. The median duration between start of chemotherapy and $1^{\text {st }}$ follow-up was 4 days, while until $2^{\text {nd }}$ follow-up was 97 days.

\subsection{Cancer Treatments}

Fourteen patients $(28 \%)$ received epirubicin, 5 patients $(10 \%)$ received trastuzumab and 2 patients $(4 \%)$ received docetaxel with mean cumulative doses of $470 \pm 80 \mathrm{mg} / \mathrm{m}^{2}$, $2550 \pm 750 \mathrm{mg}$ and $477 \pm 208 \mathrm{mg} / \mathrm{m}^{2}$, respectively.

\subsection{Changes During Follow-up}

Changes in cardiac biomarkers and CMR parameters over 3 months were evaluated through multiple comparisons between baseline and $1^{\text {st }}$ as well as $2^{\text {nd }}$ follow-up.

\subsubsection{Cardiac Biomarkers}

Compared to baseline, significant elevation of plasma NtproBNP and serum hs-TnT was found at the $1^{\text {st }}$ and $2^{\text {nd }}$ follow-up with $\mathrm{p}$ value $=0.014$ and 0.002 , respectively, see Table 2. There was no significant interaction between treatment with epirubicin and change over time of hs-TnT (p $=0.67)$ or NT-proBNP $(\mathrm{p}=0.79)$.

Table 2. Changes in cardiac biomarkers.

\begin{tabular}{|c|c|c|c|c|c|}
\hline & Baseline $(n=21)$ & $1^{\text {st }}$ Follow-up $(n=21)$ & p value & $2^{\text {nd }}$ Follow-up $(n=21)$ & p value \\
\hline hs-TnT (pg/ml) & $4(4-5.5)$ & $5(4-6.7)$ & 0.077 & $7(4.5-12.5)$ & $0.002 *$ \\
\hline Nt-proBNP (ng/l) & $131(82.5-174.5)$ & $190(111-242)$ & $0.014^{*}$ & $118(57.5-219)$ & 0.958 \\
\hline
\end{tabular}

Data are expressed as median (IQR); hs-TnT, high-sensitivity troponin T; NT-proBNP, N-terminal pro brain natriuretic peptide; p, probability; *, significance $<0.05$. P values indicate significance of comparisons with baseline using Friedman's and Wilcoxon signed-rank tests.

\subsubsection{CMR Parameters}

The mean LVEF tended to mildly decrease during followup reaching $(63.9 \% \pm 4.7)$ at $2^{\text {nd }}$ follow-up in comparison to baseline study $(65.8 \% \pm 4.7 ; \mathrm{p}=0.076)$. This change was accompanied by a mild increase of the mean left ventricular endsystolic volume index (LVESVi) from $23.3 \pm 5 \mathrm{ml} / \mathrm{m}^{2}$ at baseline reaching to $25.3 \pm 6.7 \mathrm{ml} / \mathrm{m}^{2}$ at $2^{\text {nd }}$ follow-up with $\mathrm{p}$ $=0.059$. A representative imaging example is shown in Figure $1 a, b \& c$. Moreover, a significant reduction in mean native T1 was found from $1246.6 \pm 29.5 \mathrm{~ms}$ at baseline to $1231.4 \pm 31.4 \mathrm{~ms}$ at $1^{\text {st }}$ follow-up, see Figure 2, which was followed by significant elevation at $2^{\text {nd }}$ follow-up reaching $1265.8 \pm 27.9 \mathrm{~ms}$ with $\mathrm{p}=0.011$ and 0.012 , respectively, see Figure $1 d \& e$. There was also significant elevation in mean $\mathrm{T} 2$ from $41.6 \pm 3.4 \mathrm{~ms}$ at baseline to $43.8 \pm 3.8 \mathrm{~ms}$ at $2^{\text {nd }}$ follow-up with $\mathrm{p}=0.045$, see Figure If \& $\mathrm{g}$. Out of 21 patients, only 1 patient (4.8\%) experienced cardiotoxicity as defined by an LVEF reduction of $>10 \%$ as compared with baseline. The mean individual changes in CMR functional and tissue characterization parameters as compared to baseline are listed in Table 3. There was no significant interaction between treatment with epirubicin and change over time of $\mathrm{T} 1$ or $\mathrm{T} 2$ with $\mathrm{p}=0.134$ and $\mathrm{p}=0.328$, respectively. Other parameters were within normal range at baseline and during follow-up. There was a significant correlation between changes at 3 months in LVEF and native myocardial T1 (Pearson's correlation coefficient $=-0.473, \mathrm{p}$ $=0.031$ ), see Figure 3, as well as between morphological and functional parameters measured by TTE and CMR. No significant interaction was found between the changes in serum biomarkers or CMR parameters and the co-existing factors such as the different type and doses of chemotherapy as well as the presence of previous breast or mediastinal radiotherapy.

Table 3. Changes in CMR functional and tissue characterization parameters.

\begin{tabular}{|c|c|c|c|c|}
\hline CMR parameters & $1^{\text {st }}$ Follow-up $(n=21)$ & p value & $2^{\text {nd }}$ Follow-up $(n=21)$ & p value \\
\hline LVEF (\%) & $-0.81 \pm 3.91$ & 0.354 & $-1.86 \pm 4.55$ & 0.076 \\
\hline $\operatorname{LVESVi}\left(\mathrm{ml} / \mathrm{m}^{2}\right)$ & $0.43 \pm 3.59$ & 0.523 & $2.05 \pm 4.69$ & 0.059 \\
\hline Native myocardial T1 (ms) & $-15.19 \pm 25$ & $0.011 *$ & $19.24 \pm 32$ & $0.012 *$ \\
\hline Myocardial T2 (ms) & $0.86 \pm 1.93$ & 0.166 & $2.24 \pm 2.74$ & $0.045^{*}$ \\
\hline
\end{tabular}

Data are expressed as mean of individual change in comparison to the baseline \pm SD; LVEF, left ventricular ejection fraction; LVESVi, left ventricular endsystolic volume index, $\mathrm{p}$, probability; ${ }^{*}$, significance $<0.05$. P values indicate significance through adjusted (Bonferroni) pairwise comparisons with baseline. 


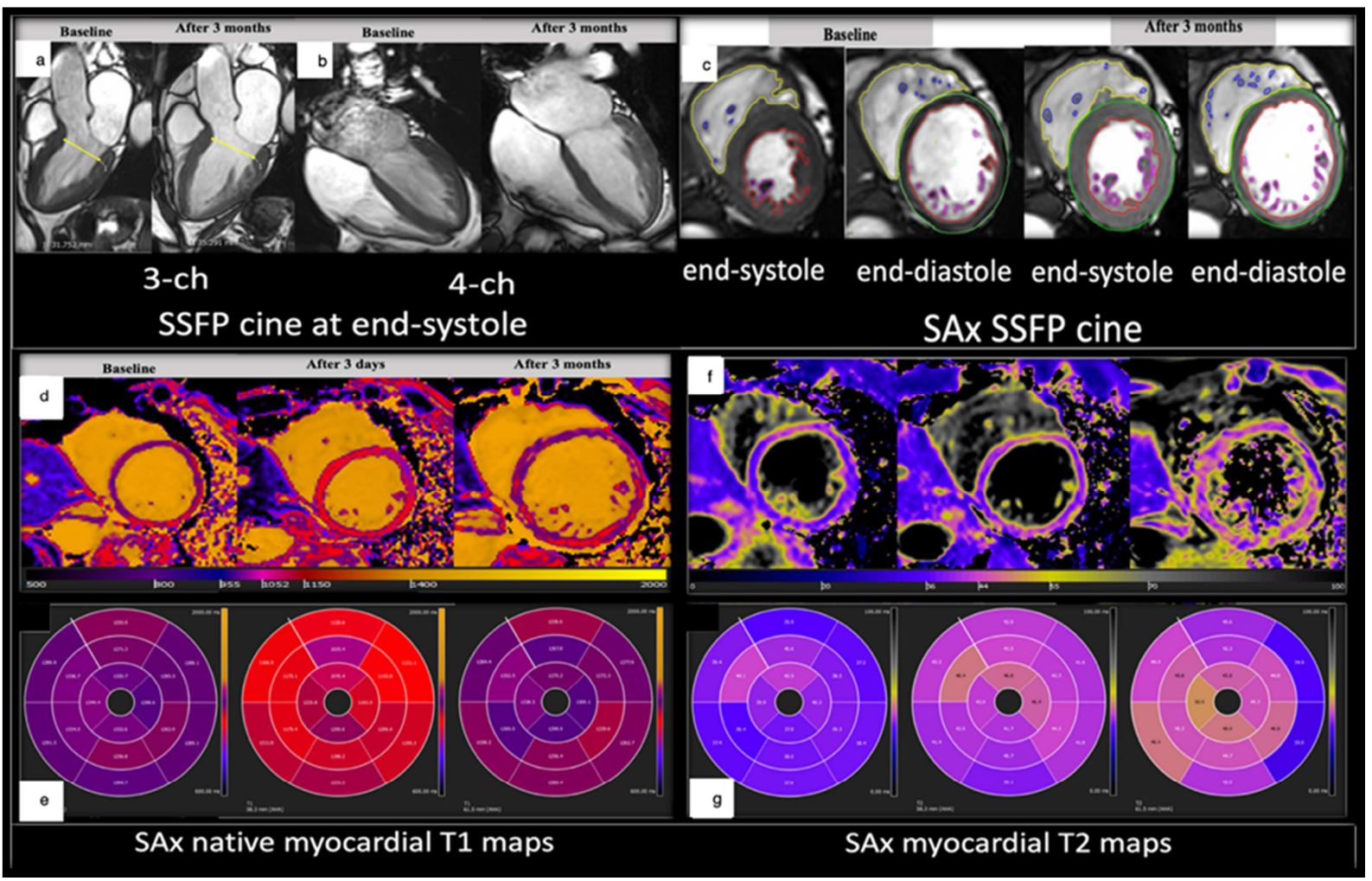

Figure 1. Representative images of trastuzumab-induced cardiotoxicity. Cine 3-chamber (a) and 4-chamber views (b) as well as short axis view (c) during baseline study $(L V E F=66 \%)$ and 3 months thereafter $(L V E F=54 \%)$. Native $T 1$ (d) showed reduction of global left ventricular value after 3 days (baseline $=$ $1196 \mathrm{~ms}, 1^{\text {st }}$ follow-up $=1172 \mathrm{~ms}$ and $2^{\text {nd }}$ follow-up $=1277 \mathrm{~ms}$ ). Myocardial T2 (f) showed subtle elevation of global value after 3 months (baseline = $40 \mathrm{~ms}$, $1^{\text {st }}$ follow-up $=43 \mathrm{~ms}$ and $2^{\text {nd }}$ follow-up $\left.=44 \mathrm{~ms}\right)$. The average segmental $T 1$ and T2 times are displayed as 'bull's eye' images (e) \& $(\mathrm{g})$, respectively. The color maps represent continuous $T 1$ and T2 values. LVEF, left ventricular ejection fraction.

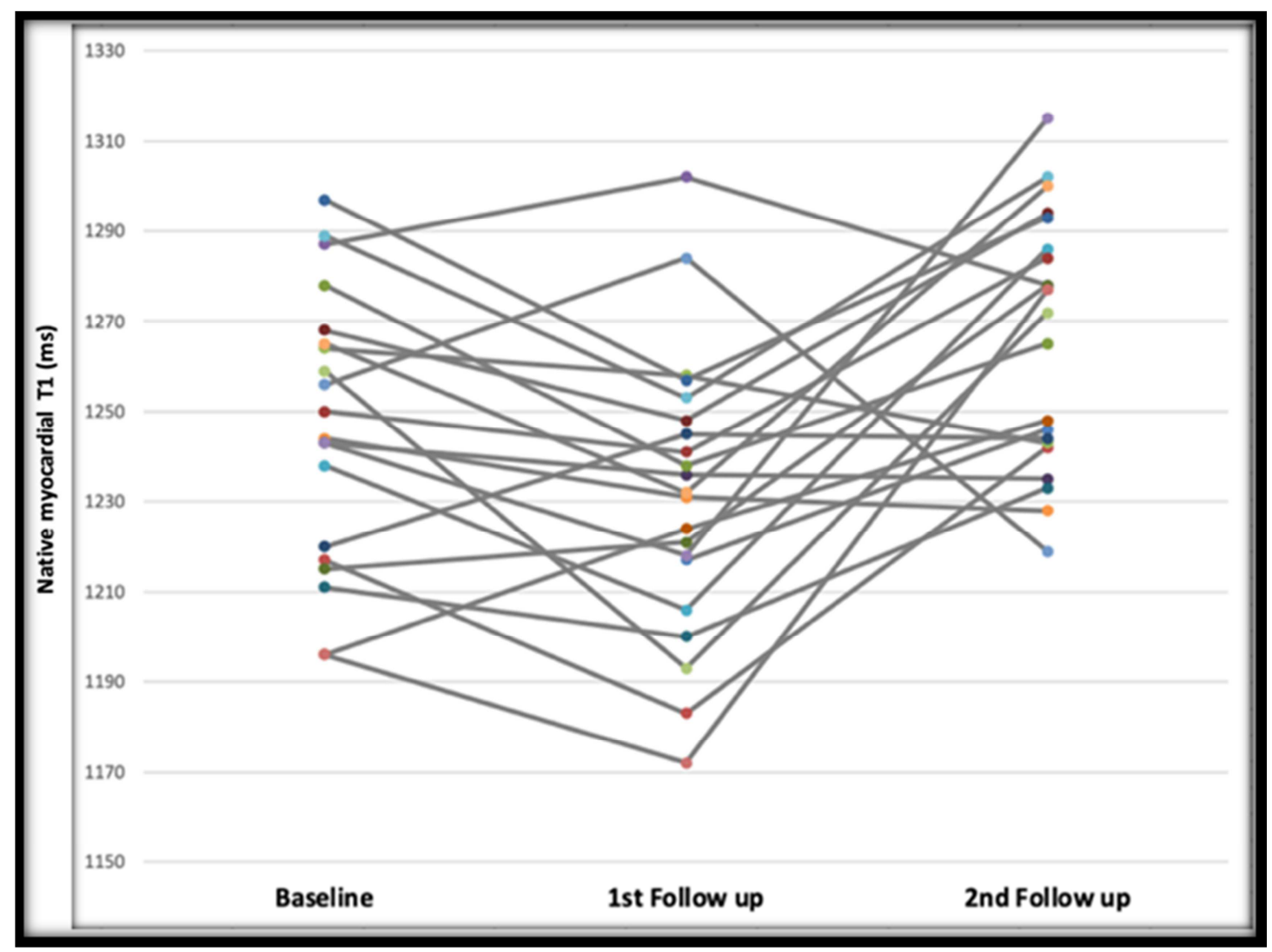

Figure 2. Individual changes of native T1 over the follow-up period. 


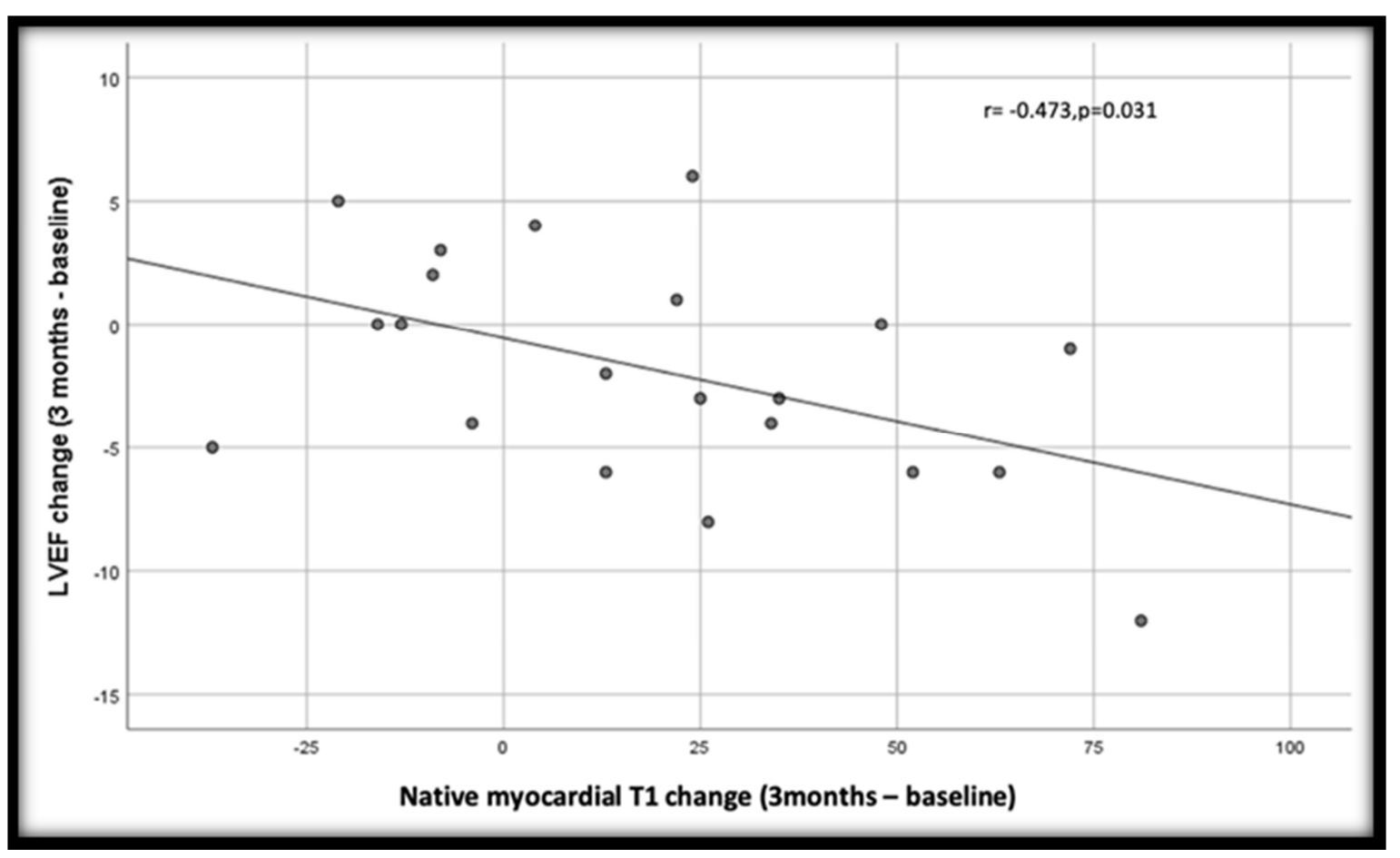

Figure 3. Relationship between changes in LVEF (\%) and native T1 (ms) over 3 months.

\section{Discussion}

Our study shows that treatment with potentially cardiotoxic drugs are associated with changes of myocardial T1 as a CMR-derived biomarker. These occur even in the absence of an overt cardiotoxic reaction in a short time follow-up of 3 month.

Over the follow-up period, we found a mild yet significant increase of cardiac biomarkers including plasma NT-proBNP and serum hs-TnT. Other studies also reported that serum hsTnT reaches its peak during anthracycline therapy. Early elevation of serum hs-TnT with trastuzumab therapy may indicate a worse prognosis [11]. In contrast, no significant change was previously reported in plasma NT-proBNP during trastuzumab [8] or anthracycline therapy [12].

CMR is playing an increasingly important role in the assessment of cardiotoxicity [13]. It has many advantages such as the lack of ionizing radiation and the ability of noninvasive tissue characterization. CMR is considered the standard reference for cardiac function evaluation [14]. In our study, the LVEF tended to mildly decrease during followup which was associated with mild increase of the mean LVESVi. This finding is consistent with another study that followed 53 cancer patients treated with anthracycline and showed that LVEF and LVESV start deteriorating as early as one month and remain abnormal 6 months after therapy initiation [12]. Another study, conducted on breast cancer patients treated with trastuzumab, also found that LVEF started to decrease after 3 months, followed by a significant reduction after 6 months [15]. In our study, the follow-up started as early as 3-6 days after chemotherapy onset and thus we could confirm an early decrease in LVEF.

Relatively recently, CMR myocardial mapping has been increasingly used for myocardial tissue characterization. This approach allows for quantitatively assessing diffuse myocardial tissue alteration [9]. Moreover, the presence of LGE reflects the presence of irreversible myocardial damage [1]. In this study, no LGE was found at baseline which suggests the lack of pre-existing myocardial scar.

In our study, the tissue characterization parameters (native $\mathrm{T} 1$ and T2) showed significant changes during follow-up. There was a significant reduction in native T1 3-6 days after chemotherapy onset, which was followed by significant increase after 3 months. This early decrease in native T1 was previously reported as a decrease of native T1 48 hours after the $1^{\text {st }}$ dose of anthracyclines. In contrast to our study this pehnomenon was only observed in patients with acute cardiotoxicity [9]. The relatively late increase in native T1 was also reported by Haslbauer et al. who reported a significant increase in native T1 within 3 months after chemotherapy initiation and remained elevated until $>12$ months. They concluded that native $\mathrm{T} 1$ is the most effective predictor of chemotherapy-induced cardiotoxicity. The same group also found a significant elevation in T2 within 3 months after chemotherapy start which was followed by later recovery [16]. This observation is similar to our findings, as we found significant increase in T2 3 months after chemotherapy initiation. Significant elevation in T2 was also reported by Galán-Arriola and colleagues 6 weeks after intracoronary doxorubicin injection in twenty pigs and considered it as the earliest marker of anthracycline-induced cardiotoxicity [17]. The mechanism of the initial native T1 reduction is still unclear. A reduced native $\mathrm{T} 1$ is observed with intracellular accumulation of iron or lipids [18]. An increased native $\mathrm{T} 1$ indicates presence of myocardial edema or diffuse fibrosis. An increased T2 is useful for the 
diagnosis of acute inflammatory processes such as myocarditis or acute infarction [19]. One recognized hypothesis of anthracyclines-related cardiotoxicity includes the formation of anthracycline-iron complexes. Moreover, it was also proposed that anthracyclines may allow an increase in cellular levels of iron which potentiate iron-mediated oxidative stress [20]. This may explain the initial decrease in native $\mathrm{T} 1$ among our cohort.

In this study, there was no significant difference in changes of CMR parameters between the different doses of chemotherapy. This finding was previously highlighted in other study showing that anthracycline-related cardiotoxicity can occur with any given dose. Moreover, the higher risk of trastuzumab-induced cardiotoxicity was not related to the cumulative dose but was mainly observed with concomitant use of anthracyclines [3]. In our study, there was no concomitant use of both treatments.

\section{Study Limitations}

Include the small sample size and short follow-up period. Therefore, studies with larger cohorts and longer follow-up are recommended for validation of our findings. The heterogeneity of cancer treatments is also considered limitation of this study. However, the longitudinal follow-up has improved the accuracy of our results together with the absence of significant difference in CMR temporal changes regarding the different chemotherapy types and doses.

\section{Conclusion}

Our study shows that breast cancer treatment may cause early cardiac tissue alteration in the absence of significant reduction of ventricular systolic performance. CMR derived myocardial mapping, specifically the early decrease of native T1 times may enable early identification of cardiotoxicity among breast cancer patients treated with anthracyclines or trastuzumab and thus may allow identification of patients at risk. However, further studies with larger cohorts and longer follow-up are recommended for validation of our findings.

\section{Conflict of Interest}

The authors declare that they have no competing interests.

\section{Ethical Standards}

This study was approved by the local ethics committee of University of Heidelberg, Germany. All enrolled patients gave written informed consent before participation.

\section{Acknowledgements}

We thank our MR technologists and study nurses especially Regula Gnirs, Daniel Helm, Melanie Feiner, Miriam Hess, Vesna Bentele, Leonie Siegmund and Ines Ludwig for their continuous support with recruitment and data acquisition. Dr N. A. Atia was supported by GERLS Scholarship (German Egyptian Research Long-term Scholarship) which was jointly funded by the Egyptian Ministry of Higher Education (MHESR) and the German Academic Exchange Service (DAAD) as well as by a graduation grant (STIBET) from DAAD.

\section{References}

[1] Ferreira de Souza T, Quinaglia T, Neilan TG, Coelho-Filho OR (2019) Assessment of Cardiotoxicity of Cancer Chemotherapy: The Value of Cardiac MR Imaging. Magn Reson Imaging Clin N Am 27 (3): 533-544. doi: 10.1016/j.mric.2019.04.001.

[2] Herrmann J, Lerman A, Sandhu NP, Villarraga HR, Mulvagh SL, Kohli M (2014) Evaluation and management of patients with heart disease and cancer: cardio-oncology. Mayo Clin Proc 89 (9): 1287-1306. doi: 10.1016/j.mayocp.2014.05.013.

[3] Adao R, de Keulenaer G, Leite-Moreira A, Bras-Silva C (2013) Cardiotoxicity associated with cancer therapy: pathophysiology and prevention strategies. Rev Port Cardiol 32 (5): 395-409. doi: 10.1016/j.repc.2012.11.002.

[4] Jordan JH, Vasu S, Morgan TM, D'Agostino RB, Jr., Melendez GC, Hamilton CA, Arai AE, Liu S, Liu CY, Lima JA, Bluemke DA, Burke GL, Hundley WG (2016) Anthracycline-Associated T1 Mapping Characteristics Are Elevated Independent of the Presence of Cardiovascular Comorbidities in Cancer Survivors. Circ Cardiovasc Imaging 9 (8). doi: 10.1161/CIRCIMAGING.115.004325.

[5] Chen-Scarabelli C, McRee C, Leesar MA, Hage FG, Scarabelli TM (2017) Comprehensive review on cardiooncology: Role of multimodality imaging. J Nucl Cardiol 24 (3): 906-935. doi: 10.1007/s12350-016-0535-y.

[6] Fallah-Rad N, Walker JR, Wassef A, Lytwyn M, Bohonis S, Fang T, Tian G, Kirkpatrick ID, Singal PK, Krahn M, Grenier D, Jassal DS (2011) The utility of cardiac biomarkers, tissue velocity and strain imaging, and cardiac magnetic resonance imaging in predicting early left ventricular dysfunction in patients with human epidermal growth factor receptor IIpositive breast cancer treated with adjuvant trastuzumab therapy. J Am Coll Cardiol 57 (22): 2263-2270. doi: 10.1016/j.jacc.2010.11.063.

[7] Shimoyama M, Murata Y, Sumi K-I, Hamazoe R, Komuro I (2001) Docetaxel induced cardiotoxicity. Heart 86 (2): 219. doi: 10.1136/heart.86.2.219.

[8] Ong G, Brezden-Masley C, Dhir V, Deva DP, Chan KKW, Chow CM, Thavendiranathan D, Haq R, Barfett JJ, Petrella TM, Connelly KA, Yan AT (2018) Myocardial strain imaging by cardiac magnetic resonance for detection of subclinical myocardial dysfunction in breast cancer patients receiving trastuzumab and chemotherapy. Int J Cardiol 261: 228-233. doi: 10.1016/j.ijcard.2018.03.041.

[9] Muehlberg F, Funk S, Zange L, von KnobelsdorffBrenkenhoff F, Blaszczyk E, Schulz A, Ghani S, Reichardt A, Reichardt P, Schulz-Menger J (2018) Native myocardial T1 time can predict development of subsequent anthracyclineinduced cardiomyopathy. ESC Heart Fail 5 (4): 620-629. doi: 10.1002/ehf2.12277. 
[10] Seidman A, Hudis C, Pierri MK, Shak S, Paton V, Ashby M, Murphy M, Stewart SJ, Keefe D (2002) Cardiac Dysfunction in the Trastuzumab Clinical Trials Experience. Journal of Clinical Oncology 20 (5): 1215-1221. doi: 10.1200/JCO.2002.20.5.1215.

[11] Cardinale D, Colombo A, Torrisi R, Sandri MT, Civelli M, Salvatici M, Lamantia G, Colombo N, Cortinovis S, Dessanai MA, Nolè F, Veglia F, Cipolla CM (2010) Trastuzumabinduced cardiotoxicity: clinical and prognostic implications of troponin I evaluation. J Clin Oncol 28 (25): 3910-3916. doi: 10.1200/jco.2009.27.3615.

[12] Drafts BC, Twomley KM, D'Agostino R, Jr., Lawrence J, Avis N, Ellis LR, Thohan V, Jordan J, Melin SA, Torti FM, Little WC, Hamilton CA, Hundley WG (2013) Low to moderate dose anthracycline-based chemotherapy is associated with early noninvasive imaging evidence of subclinical cardiovascular disease. JACC Cardiovasc Imaging 6 (8): 877 885. doi: 10.1016/j.jcmg.2012.11.017.

[13] Yu AF, Chan AT, Steingart RM (2019) Cardiac Magnetic Resonance and Cardio-Oncology: Does T2 Signal the End of Anthracycline Cardiotoxicity? J Am Coll Cardiol 73 (7): 792794. doi: 10.1016/j.jacc.2018.11.045.

[14] Meyersohn NM, Pursnani A, Neilan TG (2015) Detection of Cardiac Toxicity Due to Cancer Treatment: Role of Cardiac MRI. Curr Treat Options Cardiovasc Med 17 (8): 396. doi: 10.1007/s11936-015-0396-8.

[15] Nakano S, Takahashi M, Kimura F, Senoo T, Saeki T, Ueda S, Tanno J, Senbonmatsu T, Kasai T, Nishimura S (2016) Cardiac magnetic resonance imaging-based myocardial strain study for evaluation of cardiotoxicity in breast cancer patients treated with trastuzumab: A pilot study to evaluate the feasibility of the method. Cardiol J 23 (3): 270-280. doi: 10.5603/CJ.a2016.0023.

[16] Haslbauer JD, Lindner S, Valbuena-Lopez S, Zainal H, Zhou H, D'Angelo T, Pathan F, Arendt CA, Bug G, Serve H, Vogl TJ, Zeiher AM, Carr-White G, Nagel E, Puntmann VO (2019) CMR imaging biosignature of cardiac involvement due to cancer-related treatment by T1 and T2 mapping. Int J Cardiol 275: 179-186. doi: 10.1016/j.ijcard.2018.10.023.
[17] Galan-Arriola C, Lobo M, Vilchez-Tschischke JP, Lopez GJ, de Molina-Iracheta A, Perez-Martinez C, Aguero J, Fernandez-Jimenez R, Martin-Garcia A, Oliver E, VillenaGutierrez R, Pizarro G, Sanchez PL, Fuster V, SanchezGonzalez J, Ibanez B (2019) Serial Magnetic Resonance Imaging to Identify Early Stages of Anthracycline-Induced Cardiotoxicity. J Am Coll Cardiol 73 (7): 779-791. doi: 10.1016/j.jacc.2018.11.046.

[18] Messroghli DR, Moon JC, Ferreira VM, Grosse-Wortmann L, He T, Kellman P, Mascherbauer J, Nezafat R, Salerno M, Schelbert EB, Taylor AJ, Thompson R, Ugander M, van Heeswijk RB, Friedrich MG (2017) Clinical recommendations for cardiovascular magnetic resonance mapping of $\mathrm{T} 1, \mathrm{~T} 2$, T2* and extracellular volume: A consensus statement by the Society for Cardiovascular Magnetic Resonance (SCMR) endorsed by the European Association for Cardiovascular Imaging (EACVI). J Cardiovasc Magn Reson 19 (1): 75. doi: 10.1186/s12968-017-0389-8.

[19] Loffler AI, Salerno M (2018) Cardiac MRI for the evaluation of oncologic cardiotoxicity. J Nucl Cardiol 25 (6): 2148-2158. doi: $10.1007 / \mathrm{s} 12350-018-1293-9$.

[20] Gammella E, Maccarinelli F, Buratti P, Recalcati S, Cairo G (2014) The role of iron in anthracycline cardiotoxicity. Front Pharmacol 5: 25. doi: 10.3389/fphar.2014.00025.

\section{Biography}

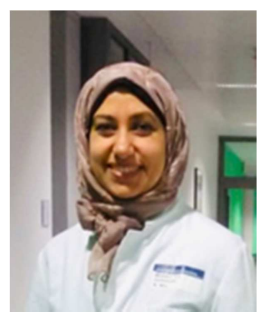

Noura Abdelmonem Atia, M. B. B. Ch., MSc. Assistant lecturer at the department of Diagnostic Radiology, Faculty of Medicine, Tanta University, Tanta, Egypt. Research fellow at the department of Cardiology, Angiology and Pulmonology, Internal Medicine III, Heidelberg University Hospital, Heidelberg, Germany. ORCID iD: https://orcid.org/0000-0003-0908-4397 